\title{
Towards the thermodynamics of localization processes
}

\author{
Paolo Grigolini, ${ }^{1,2,3}$ Marco G. Pala, ${ }^{3}$ Luigi Palatella, ${ }^{3,4}$ and Roberto Roncaglia ${ }^{4}$ \\ ${ }^{1}$ Istituto di Biofisica CNR, Area della Ricerca di Pisa, Via Alfieri 1, San Cataldo 56010 Ghezzano-Pisa, Italy \\ ${ }^{2}$ Center for Nonlinear Science, University of North Texas, P.O. Box 5368, Denton, Texas 76203-5368 \\ ${ }^{3}$ Dipartimento di Fisica dell'Università di Pisa and INFM, Piazza Torricelli 2, 56127 Pisa, Italy \\ ${ }^{4}$ Istituto Nazionale per la Fisica della Materia, UdR di Pisa, Via Buonarroti 2, 56126 Pisa, Italy
}

(Received 29 July 1999; revised manuscript received 25 January 2000)

\begin{abstract}
We study the entropy time evolution of a quantum mechanical model, which is frequently used as a prototype for Anderson's localization. Recently Latora and Baranger [Phys. Rev. Lett. 82, 520 (1999)] found that there exist three entropy regimes, a transient regime of passage from dynamics to thermodynamics, a linear-in-time regime of entropy increase, that is, a thermodynamic regime of Kolmogorov kind, and a saturation regime. We use the nonextensive entropic indicator advocated by Tsallis [J. Stat. Phys. 52, 479 (1988)] with a mobile entropic index $q$, and we find that the adoption of the "magic" value $q=Q=1 / 2$, compared to the traditional entropic index $q=1$, reduces the length of the transient regime and makes earlier the emergence of the Kolmogorov regime. We adopt a two-site model to explain these properties by means of an analytical treatment and we argue that $Q=1 / 2$ might be a typical signature of the occurrence of Anderson localization.
\end{abstract}

PACS number(s): 05.45.Mt, 05.20.-y, 03.65.Bz

\section{INTRODUCTION}

In this paper we focus our attention on the process of localization discovered by Anderson [1,2], and we discuss the corresponding time evolution using the nonextensive thermodynamics view of Tsallis $[3,4]$. The subject of Tsallis nonextensive thermodynamics is attracting the interest of an ever increasing number of investigators in different branchs of complexity theory (see, for instance, Ref. [5]). We want to apply this new perspective to the delicate problem of the connection between thermodynamics and quantum dynamics.

According to the new paradigm of deterministic chaos, study of this connection leads to study of the quantum behavior of those systems that would be chaotic in the classical limit. The subject of the entropy increase of quantum systems that would be classically chaotic has been addressed in a number of papers [6-9]. The pioneering work of Ref. [6] has established that the coarse graining necessary for the entropy to increase can be produced by a weak fluctuation. As a consequence of this, after a transient of time duration inversely proportional to the classical Lyapunov coefficient the system is expected to reach a thermodynamic condition. This prediction is based on several conjectures. The first is that there must be a kind of equivalence between the Gibbs entropy and the Kolmogorov-Sinai (KS) entropy $[10,11]$ $h_{\mathrm{KS}}$. The Gibbs entropy is a functional of probability density, whereas $h_{\mathrm{KS}}$ is the entropy of a trajectory and, in principle, might not coincide with the entropy expressed in terms of probability density. Furthermore, the KS entropy is an entropy per unit time and the existence of a finite value of $h_{\mathrm{KS}}$ implies a steady rate of entropy increase. Consequently, one must make a second important conjecture: The thermodynamic regime corresponds to the probability entropy increasing as a linear function of time.

Both conjectures are supported by the recent findings of Latora and Baranger [12]. These authors studied several chaotic maps and found that the time evolution of the Gibbs entropy goes through three time regimes: (i) an early regime of exponential increase, (ii) an intermediate time regime of linear increase, the Kolmogorov regime, and, finally, (iii) a saturation regime. On the basis of arguments similar to those used here earlier, the second time regime is identified with the thermodynamic regime. In this classical case, the coarse graining is done by the division of the space into cells.

The quantum case [6-9] is very complex. The results depend on the relations among three fundamental parameters, expressed in the same units. These are $\hbar, I$, and $D$. The first is the Planck constant, the second the classical action, and the third the intensity of the coarse grain generating stochastic force. For the quantum system to exhibit ordinary thermodynamic behavior it is necessary not only that $\hbar \ll I$ but also that $\hbar<D[6]$. In this case the rate of increase of the von Neumann entropy is found [8] to be proportional to the KS entropy.

The authors of Ref. [7] focused their attention on the transition from dynamics to thermodynamics, namely, the first of the three time regimes discussed in Ref. [12], in the case of the quantum Arnold's cat [8]. They focused their attention on the condition $I \gg D \gg \hbar$, where, according to [6], an exponential-like transition from dynamics to thermodynamics is expected to occur. The numerical results of these authors provide a satisfactory support to the heuristic arguments of Zurek and Paz [6]. The authors of Ref. [9] studied the quantum kicked rotor in the regime where $\hbar \approx D$, and found that it yields an entropy increase proportional to $t^{\alpha}$, with $\alpha>1$. This finding agrees with the result found by Pattanayak and Brumer [7] in the case of the quantum Arnolds's cat, when the same condition $\hbar \approx D$ applies. The authors of Ref. [7], however, overlook this interesting aspect, probably because an algebraic entropy increase in time is interpreted as a dramatic postponement of the transition to thermodynamics. The authors of Ref. [9], on the contrary, found that in this case also a kind of thermodynamic behavior, namely, a regime of linear increase in time, shows up quickly when they adopt a nonextensive form of entropy indicator. 
According to Tsallis [3] the proper entropy indicator, expressed in terms of the quantum statistical density $\rho$, reads

$$
S_{q}(t) \equiv \frac{1-\operatorname{Tr}\left\{\rho^{q}(t)\right\}}{q-1} .
$$

It is straightfoward to show [3] that at $q=1$ the entropy indicator of Eq. (1) becomes identical to the Gibbs entropy indicator. Note that $q$ is thought of as a variable parameter, implying that we make the observation of the entropy time evolution for different values of $q$ until we detect the "magic value" of $q$, denoted by $Q$, as the value of the entropic index establishing again the three regimes discovered by Latora and Baranger [12]. In the case studied in this paper the observed density matrix, as we shall see, does not undergo a unitary time evolution, thereby implying no need of an environment-induced coarse graining. As a consequence of this property, in the case of a very strong Anderson ramdomness, as we shall see, the first time regime can become so short as to become invisible on the time scale that we shall choose to illustrate the second time regime. The case of ordinary statistical mechanics corresponds to $Q=1$, namely, to the case when the Kolmogorov regime emerges from the adoption of the Gibbs entropy [12]. The departure of $Q$ from $Q=1$ signals that the dynamics of the system do not fulfill the condition of short-range interaction and correlation that according to the traditional wisdom are necessary to establish thermodynamic properties. The authors of Ref. [9] find that the entropy of Eq. (1) increases linearly in time if $q$ $=Q$, where $Q \approx 0.33$. They argued that $Q<1$ is a signature of the occurrence of Anderson localization, which is known to be a property of the quantum kicked rotor [13]. In this paper we shall deal with this issue using the statistical approach of Ref. [14]. We shall see that this implies a conflict with ordinary statistical mechanics [15].

We aim at substantiating the conjecture of Ref. [9] and for this purpose we study the Anderson tight-binding Hamiltonian. In other words, we study a prototype of Anderson localization processes, rather than the quantum kicked rotor. It is well known that the rate of energy increase of the quantum kicked rotor is made to vanish with a finite time scale by quantum correlation, namely, by the same cause as that responsible for Anderson localization [13]. However, the exact equivalence between the dynamics of the quantum kicked rotor and that of disordered solids [16] seems to be questionable [17]. This might generate doubts about the conjecture of Ref. [9] that the process of Anderson localization has a nonextensive thermodynamic nature. Thus, a direct study of the prototype model of Anderson localization would make more convincing the conjecture of Ref. [9], if we found, as we will, that in this case also the variable entropic index $q$ must be assigned a value $Q<1$ for the Kolmogorov condition, and the related thermodynamic behavior, to emerge. It has been pointed out that in the case under study in this paper recourse to environment-induced coarse graining is not necessary. This is because the entropy indicator will be expressed in terms of a proper statistical density matrix $\rho_{s}$. This statistical density, according to the prescription of an earlier paper [14], is an average over infinitely many realizations of a random distribution of site energies.
The outline of this paper is as follows. Section II defines the model under study in this paper and points out that the Anderson noise has the twofold role of creating statistical mechanics and localization. The former aspect, in an apparent conflict with the latter, is compatible with the existence of a thermodynamic perspective. Section III is devoted to the illustration of the numerical results of this paper. This numerical treatment has to be considered as a sort of exact treatment of the entropy time evolution triggered by Anderson randomness. An analytical treatment of the problem, shedding light on the reasons why the Tsallis entropy indicator is so efficient, is illustrated in Sec. IV. Finally, Sec. V is devoted to discussing the results of this paper.

\section{MASTER EQUATION, ANDERSON LOCALIZATION, AND STATISTICAL MECHANICS}

The main purpose of this section is to show that the tightbinding Hamiltonian system that we use to discuss Anderson localization is a remarkable example of joint action of randomness and order. This is, in other words, a system that is not equivalent to the classical condition of full chaos. Rather, as we shall see, this system is equivalent to the condition of weak classical chaos, or to the condition of sporadic randomness.

We study a system described by the Hamiltonian

$$
H=H_{0}+W \text {, }
$$

where

$$
H_{0} \equiv \sum_{m} E_{m}|m\rangle\langle m|
$$

and

$$
W \equiv V \sum_{m}(|m\rangle\langle m+1|+| m+1\rangle\langle m|) .
$$

This is the Hamiltonian originally taken into account by Anderson [1,2]. We make this Hamiltonian result in a transport process different from the ballistic diffusion of a perfect crystal by assuming that

$$
E_{m}=\epsilon+\phi_{m} .
$$

Here we are assuming that at different sites there is a fluctuation $\phi_{m}$ around the common value $\epsilon$. We assume no correlation among different sites, namely,

$$
\left\langle\phi_{m} \phi_{m^{\prime}}\right\rangle=A \delta_{m m^{\prime}},
$$

where $\langle\ldots\rangle$ means an average over infinitely many realizations of the Anderson noise.

It has to be pointed out that according to the prescriptions of quantum statistical mechanics, any entropy indicator must be expressed in terms of the density matrix associated with the Hamiltonian of Eq. (2). The time evolution of this density matrix is unitary, and consequently any form of entropic indicator, expressed in terms of the density matrix, is time independent. From this point of view, there is no difference between the system under study and a system characterized by regular dynamic properties, and thus strongly departing 
from the randomness condition intuitively associated with the second principle. This is disconcerting. At first sight, in fact, the Anderson prescriptions of Eqs. (5) and (6) sow a seed of randomness into the system dynamics and the entropy indicator should make this randomness ostensible. So the question is raised of how to make the entropy indicator sensitive to this randomness.

The source of entropy increase in the model of Zurek and Paz [6] is given by the deterministic chaos that the system would exhibit in the classical limit. To trigger a regime of entropy increase, these authors took into account the influence of the environment as a source of dephasing, a process that does not imply any exchange of energy between the system and its environment. This is in line with the second law which forces entropy to increase (or to remain constant if the process is reversible) if no thermal exchange with the environment is allowed. This means that an interaction between system and environment is allowed, provided that it does not cause any energy exchange. The perspective of Zurek and Paz is not trivial, because, even if the entropy increase is made possible by the key ingredient of external fluctuations, these are so weak that the time scale of the process of transition from dynamics to thermodynamics is determined by the Lyapunov coefficients, namely, a genuinely dynamic property of the Hamiltonian system under study.

Here we find that the Anderson randomness is a sort of counterpart of the deterministic chaos randomness of Zurek and Paz [6]. To reveal this randomness we must adopt the statistical density matrix defined by

$$
\rho_{s}(t) \equiv \int d[\phi] w([\phi]) \rho([\phi], t)
$$

The integrand of Eq. (7) is proportional through the statistical weighting factor $w([\phi], t)$ to $\rho([\phi], t)$, which denotes the ordinary density matrix corresponding to a given random distribution of the energy fluctuations $\phi_{m}$. The symbol $[\phi]$ denotes a given Anderson realization, that is, $[\phi]$ $\equiv \phi_{1}, \phi_{2}, \ldots, \phi_{i} \ldots$ Note that, as a consequence of the assumption of Eq. (6), we have

$$
w([\phi])=\cdots p\left(\phi_{m-1}\right) p\left(\phi_{m}\right) p\left(\phi_{m+1}\right) \cdots .
$$

We also make the assumption that the random distribution of the site energies follows the Cauchy prescription

$$
p(\phi)=\frac{1}{\pi} \frac{\gamma}{\gamma^{2}+\phi^{2}}
$$

where $p(\phi)$ denotes the probability that the energy of a given site fluctuates by the quantity $\phi$ about the common value $\epsilon$. It is interesting to remark that the time evolution of the average density matrix $\rho_{s}(t)$ of Eq. (7) becomes identical to that of a perfect lattice if $\gamma=0$. If, on the contrary, the value of the parameter $\gamma$ increases, the time evolution of the statistical density matrix $\rho_{s}(t)$ increasingly departs from the prescription of unitary time evolution. Consequently, $\gamma$, the width of the Anderson noise, can be regarded as the randomness intensity of the system.
We are therefore in a position to establish a comparison between the time evolution of $\rho_{s}(t)$ and the density matrix of the quantum kicked rotor $[8,9]$. In the latter case the source of randomness is given by the deterministic chaos of the classical time evolution of the system. In both cases, randomness has a twofold role. In the early time region of the process this randomness creates a condition of transport similar to that of ordinary Brownian motion. At later times, the diffusion process is quenched by the occurrence of Anderson localization.

The model under study rests only on the two parameters $\gamma$ and $V$, and it would be tempting for us to refer to the condition

$$
\gamma<V
$$

as weak randomness. By the same token, we are tempted to refer to the condition

$$
\gamma>V
$$

as a condition of strong randomness. This would be incorrect, since, as we shall see through the joint use of numerical calculations (Sec. III) and analytical theory (Sec. IV), in both conditions Anderson randomness and quantum correlation are present, and, the condition of Eq. (11) has the effect of realizing Anderson localization at earlier times. In a sense, this corresponds to a case where order is more significant, on the intuitive grounds that quantum correlation of larger intensity means an enhancement of the role of order. Thus, both conditions have to be considered as being equivalent to the weak chaos of classical mechanics. The case of Eq. (11) makes it possible to perform analytical calculations. Therefore in Sec. IV we shall focus on the condition of Eq. (11).

The twofold role of the Anderson randomness was studied, in the case $\gamma>V$, in an earlier publication by Mazza and Grigolini [14]. The authors of this paper proved that the time evolution of the site population $p_{n}(t) \equiv\left\langle n\left|\rho_{s}\right| n\right\rangle$ is driven by the following generalized master equation:

$$
\frac{\partial}{\partial t} p_{n}(t)=-\sum_{m \neq n} \int_{0}^{t} d t^{\prime} \Xi_{n m}\left(t-t^{\prime}\right)\left[p_{n}\left(t^{\prime}\right)-p_{m}\left(t^{\prime}\right)\right] .
$$

The authors of Ref. [14] proved that in the deep regime of strong Anderson noise $(\gamma>V)$ the memory kernel $\Xi_{n m}(t$ $-t^{\prime}$ ) becomes

$$
\Xi_{n m}(t)=2 K(t)\left(\delta_{n, m+1}+\delta_{n, m-1}\right),
$$

where

$$
\begin{aligned}
K(t)= & \frac{V^{2} \gamma}{\gamma^{2}-V^{2}} \frac{1}{\hbar^{2}}\left(\gamma e^{-2 \gamma t / \hbar}-V e^{-2 V t / \hbar}\right) \\
& +\frac{V^{3}}{\pi \gamma \hbar^{2}} \cos (2 V t / \hbar) .
\end{aligned}
$$

It is worth stressing that the oscillatory term in Eq. (14) (the second term on the right-hand side) does not play any relevant role and was introduced by the authors of Ref. [14] for the minor purpose of reproducing the weak and fast oscilla- 


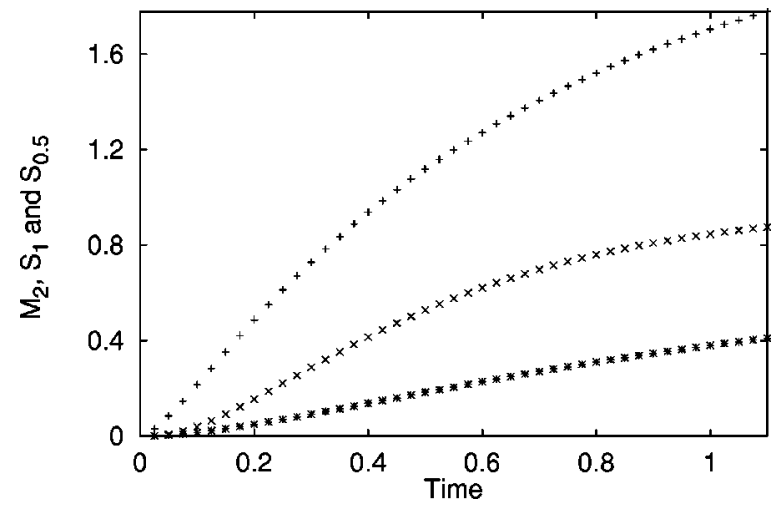

FIG. 1. The entropic indicators and the second moment of diffusion as functions of time. $\gamma / \hbar=4$ and $V / \hbar=1$. The curve denoted by + is the Tsallis entropy with $Q=0.5$; the curve denoted by $\times$ is the Gibbs entropy (the Tsallis entropy with $Q=1$ ); the curve denoted by $*$ is the second moment of the distribution, $M_{2}(t)$. Time is expressed in units of $\hbar / V$.

tions revealed by the numerical treatment. In the theoretical treatment of Sec. IV we shall make an approximation equivalent to disregarding the influence of this term.

The main result of Ref. [14] is that in the time region

$$
\frac{\hbar}{\gamma} \ll t \ll \frac{\hbar}{V}
$$

the time evolution of the system is virtually indistinguishable from ordinary Brownian diffusion. This is so because the negative and slow exponential appearing in the right-hand side of Eq. (14) is not yet strong enough to balance the fast and strong exponential. In the case $\gamma \gg V$ Anderson localization occurs at the time $\hbar / V$.

Note that the region of apparent equivalence with ordinary Brownian motion can thus be assigned an increasing time duration by keeping constant the ratio $V / \gamma \ll 1$ and decreasing the intensity of $V$. This has the effect of weakening the intensity of the negative tail of Eq. (14) and postponing the time of occurrence of Anderson localization. In Sec. IV we shall see that the entropic index $Q$ remains equal to $1 / 2$,

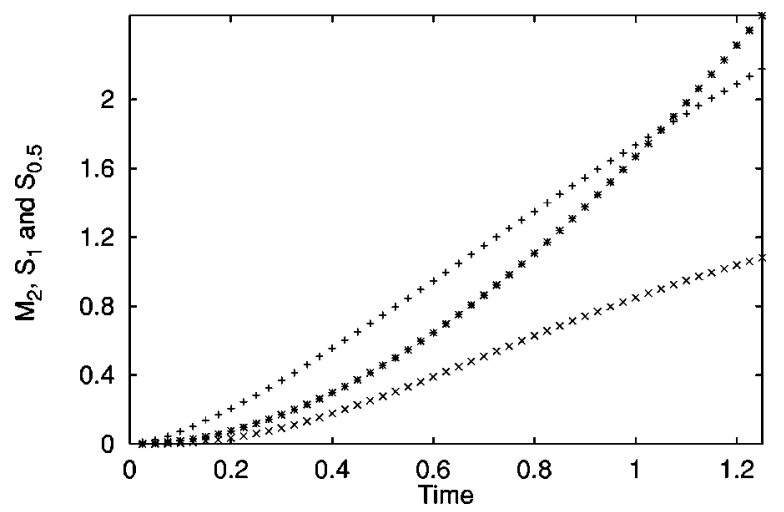

FIG. 2. The entropic indicators and the diffusion second moment as functions of time. $\gamma / \hbar=0.3$ and $V / \hbar=1$. The curve denoted by + is the Tsallis entropy with $Q=0.5$; the curve denoted by $\times$ is the Gibbs entropy (the Tsallis entropy with $Q=1$ ); the curve denoted by $*$ is the second moment of the distribution, $M_{2}(t)$. Time is expressed in units of $\hbar / V$.

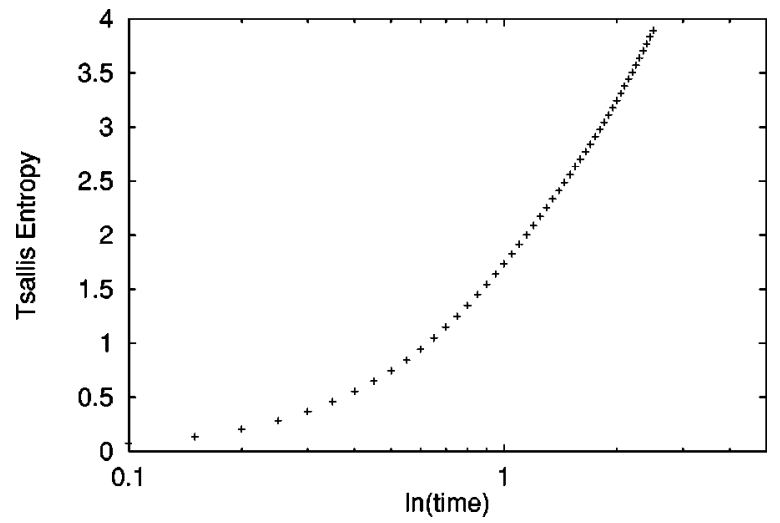

FIG. 3. The Tsallis entropy with $q=0.5$ expressed with respect to $\ln (t)$ with the same parameters as those of Fig. 2 .

regardless of the time extension of this condition, which is apparently indistinguishable from the Brownian motion regime realized with a dephasing process of external origin. In Sec. IV we shall also see that in the latter case $Q=1$. This means that the entropic index is a very sensitive indicator, actually much more sensitive than the second moment, which keeps increasing linearly in time for the whole duration of the regime of apparent Brownian motion.

\section{NUMERICAL RESULTS}

The numerical calculations have been done by producing, first of all, about 1000 realizations of the Hamiltonian system of Eqs. (2), (3), and (4). Each realization is obtained by using a random noise generator which assignes to any site $|m\rangle$ a fluctuation $\phi_{m}$ so as to realize the Cauchy prescription of Eq. (9). The Hamiltonian of each realization is diagonalized so as to determine the corresponding time evolution and the corresponding density matrix. For any realization the initial condition is given by the wave function $|\psi(0)\rangle=|m=0\rangle$. Finally, an average over all the realizations is made.

The numerical results concern these two distinct cases: (i) $\gamma \gg V$ and (ii) $\gamma \ll V$. Figure 1 refers to case (i) and Figs. 2 and 3 to case (ii). Let us examine case (i) first. Figure 1 shows the second moment of the distribution $M_{2}(t)$ $=L^{2} \Sigma_{m} m^{2} p_{m}(t)$. $L$ denotes the lattice spacing and for simplicity we assume $L=1$. We see that $M_{2}(t)$ undergoes a nonlinear time increase for a time interval of the order of $\hbar / \gamma$. After this first time region, the increase of $M_{2}(t)$ becomes linear in time. The regime of linear increase lasts for a time of the order of $\hbar / V$. In the last time regime, not shown here, the function $M_{2}(t)$ tends to become time independent, thereby signaling the occurrence of Anderson localization.

Figure 1 illustrates also the time evolution of the two entropy indicators $S_{1}(t)$ and $S_{1 / 2}(t)$, as well as that of $M_{2}(t)$. In fact, as earlier discussed in detail, we think that some interesting information can be derived from the observation of the entropy indicator

$$
S_{q}(t) \equiv \frac{1-\operatorname{Tr}\left\{\rho_{s}^{q}(t)\right\}}{q-1} .
$$

It is well known [3] that the traditional von Neumann entropy is obtained from Eq. (16) by setting $q=1$. Notice that the choice $q=Q=1 / 2$ is dictated by the theoretical argu- 


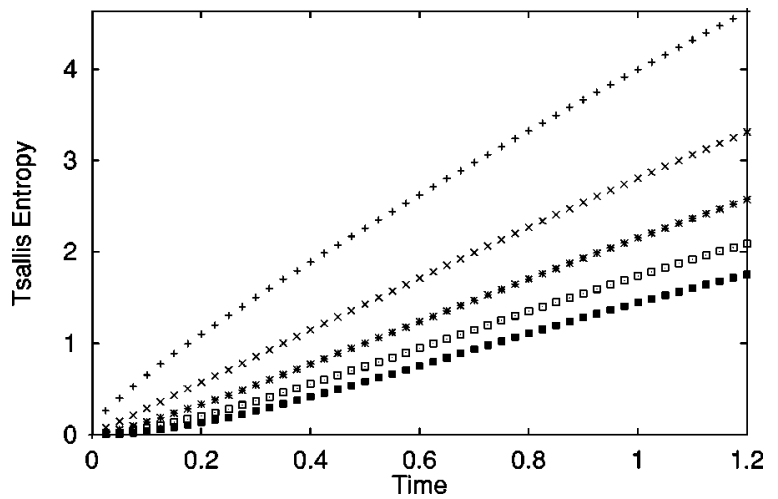

FIG. 4. The entropic indicators $S_{q}(t)$ as functions of time. The values of the parameters are $\gamma / \hbar=0.3$ and $V / \hbar=1$. The plotted curves from the bottom to the top refer to $q=0.3, q=0.4, q$ $=0.5, q=0.6$, and $q=0.7$.

ments of Sec. IV. Here we limit ourselves to pointing out that with $q=0.5$ the transition to the regime of entropy linearly increasing with time is faster than with $q=1$. We also notice that the slope of the resulting straight line is $2 \mathrm{~V} / \hbar$, in accordance with the theoretical prediction of Sec. IV. The regime of linear-in-time entropy increase, namely, the Kolmogorov regime, is not very extended in this case, the reason being that almost immediately after its onset a new transition takes place, to the stationary diffusion regime, which is characterized by the entropy increasing logarithmically in time. This is the reason why in Sec. IV the theoretical search for the magic $Q$, reflecting a genuine Kolmogorov regime, will be made at the time $\hbar / \gamma$, namely, immediately at the end of the transition regime. The time duration of the Kolmogorov regime is limited by the fast onset of the logarithmic regime, and it will become much shorter at the higher values of $\gamma$ considered in Fig. 5 below. Nevertheless, $S_{1 / 2}$ is an entropy indicator more reliable than $S_{1}$, in particular because it allows us to interpret as thermodynamic the early time region that, on the basis of $S_{1}$, would be mistaken as a regime of transition to thermodynamics. The theoretical arguments of Sec. IV will give additional support to this argument.

Figure 2 illustrates the same properties as those of Fig. 1, referred to case (ii). We see that the function $M_{2}(t)$ signals a transition to the statistical regime of Brownian diffusion at a time of the order $\hbar / V$. In the time scale explored by Fig. 2 there is no sign of the occurrence of Anderson localization, which takes place at a much later time. Even in this case the entropic analysis reveals the existence of the three regimes of Latora and Baranger [12]. The Kolmogorov regime lasts for a time of the order of $\hbar / V$ and the entropy $S_{1 / 2}(t)$ is a much more accurate indicator of the Kolmogorov regime. Even in this case the slope of $S_{1 / 2}(t)$ in the regime of linear increase is given by $2 \mathrm{~V} / \hbar$. We note that at a time of the order of $\hbar / V$ the entropy $S_{1 / 2}(t)$ makes a transition to a regime of logarithmic dependence on time as can be clearly observed in Fig. 3. Again, the adoption of $S_{1}(t)$ as entropy indicator does not establish a clear distinction betwen the regime of linear increase and that of logarithmic dependence on time.

Of some interest is also Fig. 4, which shows the time evolution of $S_{q}(t)$ for several values of the mobile entropic index $q$. We note in the first and second time regimes a pattern of curves recalling the form of a leaf. This leaf effect

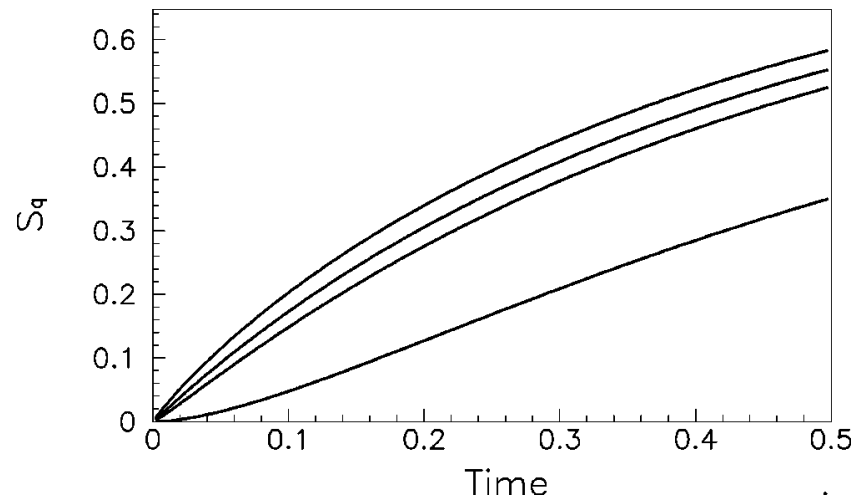

FIG. 5. The entropic indicators $S_{q}(t)$ as functions of time with Anderson randomness, $\gamma / \hbar=100$ and $V / \hbar=1$. The plotted curves from the bottom to the top refer to $q=1, q=0.6, q=0.5$, and $q$ $=0.4$.

will be discussed again in Sec. IV. We also note that this leaf effect is reminiscent of that revealed by the study of the kicked quantum rotor of Ref. [9]. The authors of Ref. [9] made the conjecture that this leaf effect might be related to the occurrence of Anderson localization. With the help of the numerical results of this section and the theoretical analysis of Sec. IV, in the concluding remarks of Sec. V we shall address this interesting issue again.

\section{NONEXTENSIVE INTERPRETATION: AN ANALYTICAL TREATMENT}

The numerical analysis of Ref. [14], suppported also by the numerical treatment of this paper, proves that the regime of strong Anderson randomness is characterized by the important fact that the transition from $m$ to $m+1$ is statistically independent of that from $m$ to $m-1$. This means that it is possible to carry out an analytical treatment based on the study of only two sites. Adoption of the distribution of Eq. (9) yields the following values for the four elements of the statistical density matrix of Eq. (7):

$$
\begin{aligned}
\rho_{s}(t)_{11}= & \int_{-\infty}^{+\infty} d E \frac{1}{\pi} \frac{2 \gamma}{4 \gamma^{2}+E^{2}} \\
& \times\left[1-\frac{4 V^{2}}{4 V^{2}+E^{2}} \sin ^{2}\left(\sqrt{E^{2}+4 V^{2}} \frac{t}{2 \hbar}\right)\right],
\end{aligned}
$$

and

$$
\begin{aligned}
\rho_{s}(t)_{12}= & \int_{-\infty}^{+\infty} d E \frac{2 i \gamma V}{\pi} \frac{1}{4 \gamma^{2}+E^{2}} \frac{1}{\sqrt{E^{2}+4 V^{2}}} \\
& \times \sin \left(\sqrt{E^{2}+4 V^{2}} \frac{t}{\hbar}\right) .
\end{aligned}
$$

Of course $\rho_{s}(t)_{21}=\rho_{s}(t)_{12}^{*}$ and $\rho_{s}(t)_{22}=1-\rho_{s}(t)_{11}$. By diagonalizing the $2 \times 2$ density matrix, we obtain the eigenvalues

$$
\Lambda_{1}(t)=\frac{1}{2}+\frac{\sqrt{\left[\rho_{s}(t)_{11}-\rho_{s}(t)_{22}\right]^{2}+4 \rho_{s}(t)_{12} \rho_{s}(t)_{21}}}{2}
$$




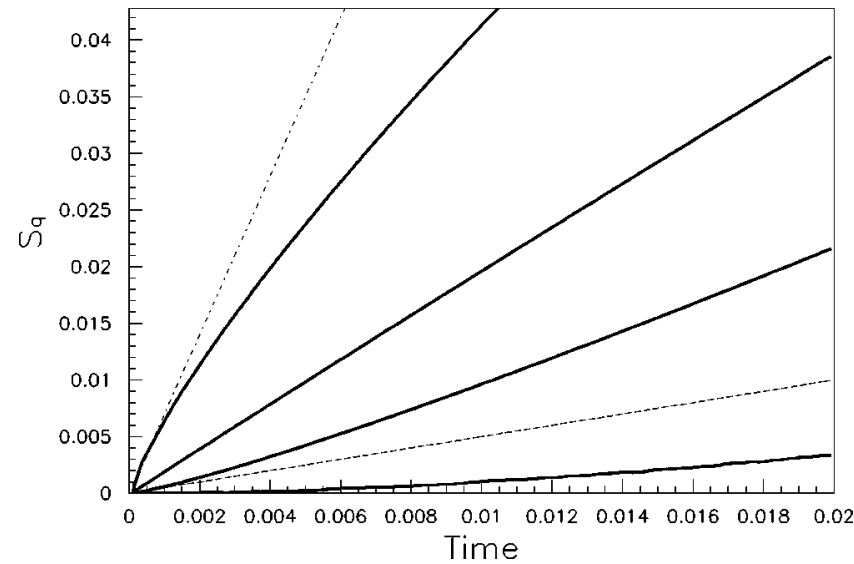

FIG. 6. The entropic indicators $S_{q}(t)$ as functions of time with Anderson randomness, $\gamma / \hbar=100$ and $V / \hbar=1$. This is nothing but an enlargement of Fig. 5. Thus, as in Fig. 5, the plotted curves from the bottom to the top refer to $q=1, q=0.6, q=0.5, q=0.4$. The dashed lines denote straight lines which are tangent to the curves at $t=0$. In the case of $q=0.5$ the tangent curve is not visible because, in the scale of this figure, it coincides with the corresponding curve. In the case of $q=1$ the tangent to the curve is not visible either because it coincides with the time axis.

and

$$
\Lambda_{2}(t)=\frac{1}{2}-\frac{\sqrt{\left[\rho_{s}(t)_{11}-\rho_{s}(t)_{22}\right]^{2}+4 \rho_{s}(t)_{12} \rho_{s}(t)_{21}}}{2} .
$$

The time evolution of the Tsallis entropy corresponding to the variable entropic index $q$ is given by

$$
S_{q}(t)=\frac{1-\Lambda_{1}(t)^{q}-\Lambda_{2}(t)^{q}}{q-1} .
$$

The expression of Eq. (21) is not yet suitable for an analytical discussion of the problem under study, since it depends on integrals defining the terms of Eqs. (17) and (18). Those integrals can be easily solved if we make the approximation of neglecting $V^{2}$ compared to $E^{2}$ in the oscillatory term of Eqs. (17) and (18). This approximation is equivalent to that of disregarding the right-hand side of Eq. (14). We thus obtain

$$
\rho_{s}(t)_{11}=1-\frac{V}{2\left(\gamma^{2}-V^{2}\right)}\left[\gamma\left(1-e^{-2 V t / \hbar}\right)-V\left(1-e^{-2 \gamma t / \hbar}\right)\right]
$$

and

$$
\rho_{s}(t)_{12}=\frac{i V}{2 \gamma}\left(1-e^{-2 \gamma t / \hbar}\right) .
$$

With the help of Eqs. (22) and (23) the time evolution of the Tsallis entropy becomes analytical. It is interesting to note that the entropy $S_{q}(t)$, plotted in Figs. 5 and 6 for different values of the entropic index $q$, results in the same kind of leaf-shape effect as that given by the numerical results of Sec. III (see Fig. 4). It is possible to prove analytically that the magic value of the mobile entropic index $q, Q$, is $Q$
$=1 / 2$. The numerical leaf-shape effect means that the adoption of the magic value of $q$ results in a linear increase of entropy as a function of time after a transient process of time duration $\hbar / \gamma$. Thus, we make an expansion of $S_{q}(t)$ supplementing the condition $V \ll \gamma$ (which is necessary to give credibility to the two-site model) with additional conditions $V t / \hbar \ll 1$ and $\gamma t / \hbar \approx 1$. We thus obtain from Eq. (21), with Eqs. (19), (20), (22), and (23),

$$
S_{q}(t) \approx \frac{1}{q-1}\left[q\left(\frac{V t}{\hbar}\right)^{2}-\left(\frac{V t}{\hbar}\right)^{2 q}\right] .
$$

It is easy to prove that the linear dependence on $t$ is obtained by assigning to $q$ the magic value $Q=1 / 2$. In fact, in this case we derive from Eq. (24)

$$
S_{1 / 2}(t) \approx-\frac{V^{2} t^{2}}{\hbar^{2}}+\frac{2 V t}{\hbar} \approx \frac{2 V t}{\hbar} .
$$

This means that the rate of entropy increase is

$$
\frac{d}{d t} S_{1 / 2}(t) \approx \frac{2 V}{\hbar} .
$$

To show that this theoretical prediction agrees with the results illustrated by Fig. 5 we refer to Fig. 6, which is obtained from Fig. 5 by enlarging the time scale. We see that on the scale of this figure the magic value $Q=1 / 2$ results in a straight line with the slope of Eq. (26), while the other values of $q$ result in a much earlier departure of the corresponding entropic indicator from the linear dependence on time.

It is interesting to compare the effects of Anderson randomness to the case where statistical mechanics are realized by environmental fluctuation. This means that, in the absence of coupling $V$ between the state $|1\rangle$ and the state $|2\rangle$, the off-diagonal matrix element between the state $|1\rangle$ and the state $|2\rangle$ would decay exponentially with the rate $2 \sigma / \hbar$, whose intensity can be thought of as the strength of the external randomness. In this condition, we would obtain a master equation identical to that of Eq. (12) with the memory kernel of Eq. (14) replaced by

$$
K(t)=\frac{V^{2}}{\hbar^{2}} e^{-2 \sigma t / \hbar} .
$$

In this specific case an analytical treatment of the same kind as that illustrated above yields an analytical expression for $S_{q}(t)$, which is then plotted in Fig. 7. It is immediately evident from Fig. 7 that in this case $Q=1$. In fact, we see that the curve corresponding to $q=1$ is a straight line. Figure 7 has to be compared to Fig. 5: This is equivalent to comparing the entropy time evolution triggered by external randomness to the entropy time evolution produced by Anderson randomness. In fact, in Fig. 5 we study the leaf effect associated with Anderson randomness with $\gamma / \hbar=100$, changing the variable entropic index $q$ from $q=1$ to $q$ $=0.4$. In Fig. 7 we do the same thing with $\sigma / \hbar=100$. We also note that the entropy increase generated by external randomness lacks the typical leaf effect of Fig. 4. In contrast, Anderson's randomness (see Fig. 5) yields a leaf effect simi- 


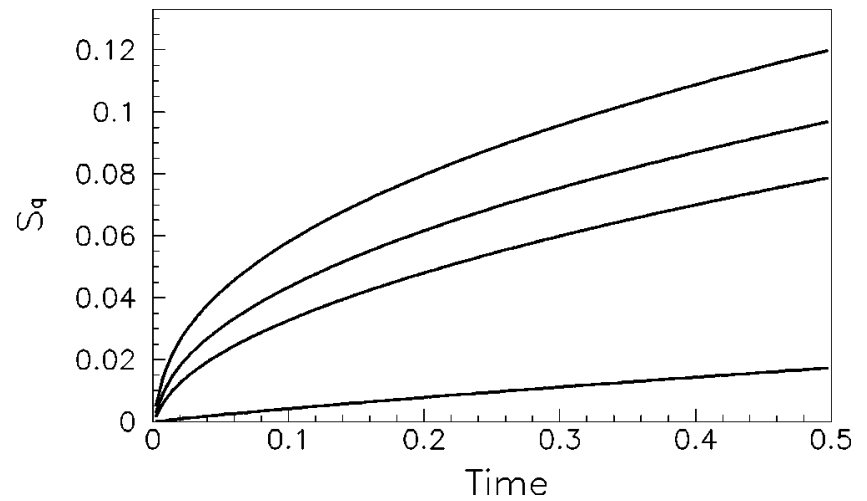

FIG. 7. The entropic indicators $S_{q}(t)$ in the presence of only external randomness as functions of time. The system's parameters are $\sigma / \hbar=100$ and $V / \hbar=1$. The plotted curves from the bottom to the top refer to $q=1, q=0.6, q=0.5$, and $q=0.4$.

lar to that of Fig. 4, the only remarkable difference being the fact that the large $\gamma$ condition strongly reduces the time duration of the first time regime.

With the help of Figs. 8 and 9 we note that in the case of merely external randomness the rate of entropy increase is proportional to $V^{2} / \sigma \hbar$. In fact, from Figs. 8 and 9 we see that the entropy rate is proportional to $1 / \sigma$ and to $V^{2}$, respectively. In other words, we find that in this case the entropy increase corresponds to the rate of the environment-induced dephasing process. This has to be contrasted with the earlier theoretical result of Eq. (26). We see, in other words, that in the case of merely external randomness the "magic" entropic index is given by the conventional entropic index $Q$ $=1$ and that the adoption of this magic entropic index reveals an ordinary source of randomness. In the case where the only source of randomness is internal, namely, Anderson randomness, the "magic" entropic index is given by the value $Q=0.5$. The corresponding nonextensive entropy is the proper entropic indicator signaling that a thermodynamic perspective is still possible in spite of dynamics dominated by quantum correlation.

\section{CONCLUDING REMARKS}

The first indisputable result of this paper is given by Figs. 1,2 , and 3 . These figures prove that the tight-binding Hamiltonian of Eq.(2) is a source of entropy time evolution clearly

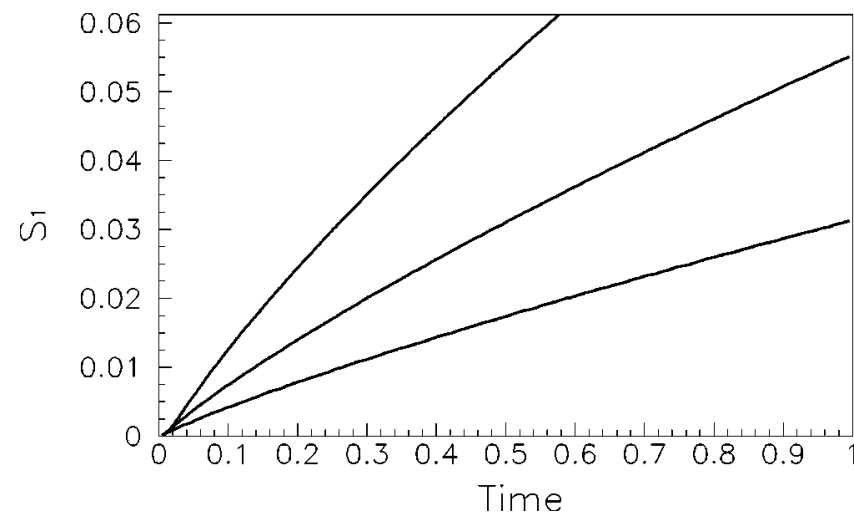

FIG. 8. Externally generated time increase of $S_{1}(t) . V / \hbar=1$. From the bottom to the top: $\sigma / \hbar=400, \sigma / \hbar=200, \sigma / \hbar=100$.

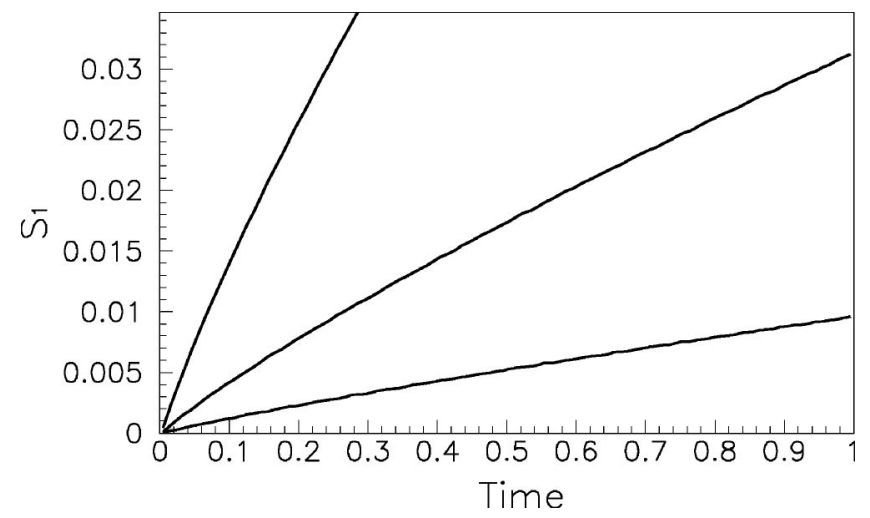

FIG. 9. Externally generated time increase of $S_{1}(t) . \sigma / \hbar=400$. From the bottom to the top: $V / \hbar=0.5, V / \hbar=1, V / \hbar=2$.

showing the three regimes recently discovered by Latora and Baranger [12]. Furthermore, from Fig. 1 we see that the regime of Brownian diffusion discussed by Mazza and Grigolini [14] is a regime of constant KS entropy.

On the basis of this observation, we would be tempted to conclude that this Kolmogorov regime is characterizecd by ordinary statistical mechanics, but this interpretation would not be totally satisfactory. In fact, it is well known [14] that this regime of apparently ordinary statistical mechanics is compatible with the silent action of quantum correlation. In the case $\gamma \gg V$ this has to do with the silent action of the negative tail of Eq. (14), whose time scale is $\hbar /(2 V)$. At the end of this transient process, Anderson localization takes place. Thus, we find it to be to some extent embarrassing to interpret this regime as a manifestation of ordinary statistical mechanics. According to the illuminating picture given by Zaslavsky in his recent book [15], ordinary statistical mechanics are closely related to deterministic motion of the type of the Bernouilli shift or map, namely, a case of dynamics with no memory. Here, on the contrary, the ensuing process of Anderson localization is a consequence of the action of quantum correlations, even if this remained silent through the whole Kolmogorov regime of Fig. 1. For this reason, we find it to be extremely interesting that the adoption of the Tsallis entropy makes a different interpretation emerge. This is so because the regime of steady entropy increase per unit time becomes clearly distinct only if we use the magic value $Q=1 / 2$, which implies that the Brownian diffusion regime studied by Mazza and Grigolini [14] is actually a form of nonextensive rather than extensive thermodynamics. This is compatible with the fact that this process is characterized by the silent action of quantum correlations which are responsible for the occurrence of Anderson localization. We note that this interpretation is also supported by the observation, numerical and analytical, that the rate of entropy increase is $2 V / \hbar$. In the case $\gamma \gg V$ this is also the rate of establishment of Anderson localization.

The results illustrated by Fig. 2 are an exciting confirmation of the fact that the magic value $Q=0.5$ results in a marked regime of linear entropy increase. However, in this case the onset of Anderson localization takes place at a time scale much larger than $\hbar / V$, and this makes it difficult to maintain the claim about a direct connection between $Q<1$ and Anderson localization. We think that in this specific case the frequency $V / \hbar$ has to do with the coherent motion of a 
regular lattice. The memory kernel $K(t)$ of Eq. (13) undergoes many oscillations with frequencies of this order of magnitude before producing localization. Thus, in this specific case the connection between $Q<1$ and Anderson localization seems to be much less direct.

Is it possible that $Q<1$ might signal quantum coherent motion, without necessarily implying Anderson localization? We think that some more research work must be devoted to this intriguing issue. The results of this paper support the conjecture of Ref. [9] that $Q<1$ is a signature of localization. We hope that this result might trigger further research to prove, or disprove, this interesting conjecture. A promising research direction is that based on the hypothesis that there exists a statistical equivalence between fractal dynamics and Anderson randomness. The result of the recent research work of Ref. [18] encourages us to pursue a search in that direction. In fact, the authors of this paper show that the logistic map at the transition to chaos, a physical condition that is known to yield $Q<1$, results in a process of localization taking place with a finite time scale. From within the context of classical dynamics the demonstration that $Q<1$ implies localization might be more direct, since $Q<1$ dictates the form of sensitivity to initial conditions [19] and this, in turn, might determine the antidispersive correlation function which is proven [18] to be responsible for localization.

Although the conjecture that $Q<1$ is closely related to Anderson localization is not yet proved, it cannot be easily dismissed either. From the theory of Sec. IV we see that if Anderson randomness vanishes and entropy increase rests only on external fluctuations, the magic entropic index $Q$ is given the ordinary value $Q=1$ again. On top of that, the rate of entropy increase is $V^{2} / \sigma \hbar$, in accordance with the fact that entropy measures randomness, and that the rate of decoherence is a proper measure of a system's randomness. Thus, we conclude that the adoption of Tsallis entropy makes it possible to adopt a thermodynamics perspective, even when the entropy rate signals a coherent property, $2 V / \hbar$, rather than an incoherent dephasing process, and that this is possible due to the adoption of the entropic index $Q=0.5$.
[1] P.W. Anderson, Phys. Rev. 109, 1492 (1958).

[2] P. W. Anderson, Rev. Mod. Phys. 50, 191 (1978).

[3] C. Tsallis, J. Stat. Phys. 52, 479 (1988).

[4] C. Tsallis, Braz. J. Phys. 29, 1 (1999).

[5] See http://tsallis.cat.cbpf.br/biblio.htm for a regularly updated bibliography on the subject.

[6] W.H. Zurek and J.P. Paz, Phys. Rev. Lett. 72, 2508 (1994).

[7] A.K. Pattanayak and P. Brumer, Phys. Rev. Lett. 79, 4131 (1997).

[8] P.A. Miller and S. Sarkar, Nonlinearity 12, 419 (1999).

[9] L. Palatella and P. Grigolini, e-print quant-ph/9810083.

[10] A.N. Kolmogorov, Dokl. Akad. Nauk SSSR 3 (1958) [Sov. Phys. Dokl. 119, 861 (1958)].

[11] Ya.G. Sinai, Dokl. Akad. Nauk SSSR 4, 768 (1959) [Sov. Phys. Dokl. 124, 769 (1959)].
[12] V. Latora and M. Baranger, Phys. Rev. Lett. 82, 520 (1999).

[13] F.M. Izrailev, Phys. Rep. 196, 299 (1990).

[14] A. Mazza and P. Grigolini, Phys. Lett. A 238, 169 (1998).

[15] G.M. Zaslavsky, Physics of Chaos in Hamiltonian Systems: on the Foundations of Statistical Physics (Imperial College Press, London, 1998).

[16] A. Altland and M.R. Zirnbauer, Phys. Rev. Lett. 77, 4536 (1996).

[17] G. Casati, F.M. Izrailev, and V.V. Sokolov, Phys. Rev. Lett. 80, 640 (1998).

[18] L. Fronzoni, P. Grigolini, and S. Montangero, Chaos Solitons Fractals (to be published).

[19] C. Tsallis, A.R. Plastino, and W.-M. Zheng, Chaos, Solitons and Fractals 8, 885 (1997). 